\title{
Ocular Manifestations of Granulomatosis with Polyangiitis: A Review of the Literature
}

Evdokia Sfiniadaki - Ioanna Tsiara - Panagiotis Theodossiadis • Irini Chatziralli

Received: January 13, 2019 / Published online: March 15, 2019

(C) The Author(s) 2019

\section{ABSTRACT}

Granulomatosis with polyangiitis (GPA) is a rare systemic autoimmune disease of unknown etiology that is characterized by granulomatous inflammation, tissue necrosis, and vasculitis in small- and medium-sized vessels. Ocular and orbital manifestations are common in almost half of patients with GPA, affecting every structure of the eye, from the eyelid and orbit to the retina, the choroid, and the optic nerve, with a wide range of severity. Since imaging findings are not always specific for the diagnosis of GPA, biopsy is useful to confirm the diagnosis. Regarding treatment, a localized pharmaceutical and surgical approach may be helpful to achieve remission, while immunosuppressive therapy, corticosteroids, and cyclosporine are also useful. In any case, multidisciplinary

Evdokia Sfiniadaki and Ioanna Tsiara contributed equally to this manuscript.

Enhanced Digital Features To view enhanced digital features for this article go to https://doi.org/10.6084/ m9.figshare.7798676.

E. Sfiniadaki · I. Tsiara

Medical School, National and Kapodistrian

University of Athens, Athens, Greece

P. Theodossiadis · I. Chatziralli $(\bowtie)$

2nd Department of Ophthalmology, National and Kapodistrian University of Athens, Athens, Greece e-mail: eirchat@yahoo.gr intervention is required to reduce the rates of relapse and morbidity in patients with GPA.

Keywords: Eye; Diagnosis; Ocular; Orbital; Treatment; Wegener's

\section{INTRODUCTION}

Granulomatosis with polyangiitis (GPA), formerly known as Wegener's granulomatosis (WG), is a rare systemic autoimmune disease of unknown etiology that occurs predominantly in Caucasians and is extremely rare in black populations [1]. The prevalence of GPA is estimated to be 3 cases per 100,000 people, and the annual incidence is approximately 8-10 cases per million, but this varies depending on location [1]. The disease was first described in 1931 by Heintz Klinger, but was named in 1936 by a German pathologist, Friedrich Wegener, who defined the disorder and clearly distinguished it from other inflammatory diseases [2]. GPA is characterized by granulomatous inflammation, tissue necrosis, and variable degrees of vasculitis in small- and medium-sized blood vessels [2].

Granulomatosis with polyangiitis has a wide spectrum of clinical manifestations with variable severity. In order to facilitate the classification of patients, GPA may be broadly divided into a limited form and a systemic form. The limited form is characterized by the absence of 
renal involvement and is isolated to the upper and lower respiratory tracts. The systemic form may involve kidneys and other organs besides the respiratory tract. Thus, patients with the systemic form suffer a more severe course of the disease compared to those with the limited form [2-4]. There is no gender predilection for the disease, although women are more likely to have the limited form of the disease [3]. Patients are typically in their fourth or fifth decade at the time of diagnosis, and fewer than $15 \%$ of all cases involve those under 19 years old [2-4].

Ocular and orbital manifestations are common in almost half of patients with either the limited or the systemic form of GPA, and they can be also present as initial features of the disease [4]. In light of the above, the purpose of this review is to shed light into the ocular manifestations of GPA while also emphasizing its pathogenesis.

This article is based on previously conducted studies and does not contain any studies with human participants or animals performed by any of the authors.

\section{PATHOGENESIS}

Although the pathogenesis of GPA remains unknown, attempts to identify its etiology have been made and have focused on the roles of genetic, immunologic, and environmental factors as well as microbial pathogens [5].

The predominance of GPA in Caucasians may indicate that genetic factors contribute to the pathogenesis of the disease. Moreover, GPA has been observed in siblings, and higher frequencies of certain HLA markers have been identified without presenting a consistent relationship with the disease $[5,6]$. Other responsible gene pathways include alpha- 1 antitrypsin deficiency and polymorphisms of CTLA-4 (which is involved in T-cell activation) and $\mathrm{Fc} \gamma$ receptor IIIb on the surfaces of neutrophils and monocytes/macrophages. Furthermore, the presence of the PTPN22 R620W allele (associated with T-cell activation) and SERPINA1 gene variants has also been implicated in GPA $[7,8]$.

The interaction between cellular and humoral immunity is believed to contribute to the disease process. Antineutrophil cytoplasmatic antibody (ANCA), either c-ANCA or p-ANCA, is associated with the appearance of GPA. In particular, c-ANCA against proteinase 3 (PR3-ANCA) is present in over $90 \%$ of patients with the disease, while the p-ANCA pattern (typically caused by antibodies against myeloperoxidase, MPO) occurs in approximately $10 \%$ of patients. The presence of ANCA stimulates neutrophil degranulation, leading to the release of lytic enzymes and reactive oxygen species, which damage the endothelium and provoke vasculitis. B cells and T cells are also involved in the pathogenesis of vasculitis. There is a strong association of granulomatous inflammation with T-cell response $[5,9]$.

Environmental etiologic factors that have been associated with GPA include dust inhalation, exposure to silica, pollution, smoking, metals, chemicals, and medicines such as hydralazine, phenytoin, sulfasalazine, antithyroid medication, and allopurinol. In addition, a role of microbes in the pathogenesis of the disease is demonstrated by the fact that chronic nasal carriage of $S$. aureus is connected with relapses of GPA [5].

\section{OCULAR MANIFESTATIONS OF GRANULOMATOSIS WITH POLYANGIITIS}

Ocular and orbital involvement can occur in patients with GPA, affecting every structure of the eye from the eyelid and orbit to the optic nerve, with a wide range of severity [4].

\section{Orbit}

Orbital involvement is one of the most frequent manifestations of GPA, occurring in almost $45-50 \%$ of patients. It may be the result of contiguous disease in adjacent paranasal sinuses, or it may occur as a focal disease with orbital granuloma formation or vasculitis [4]. Orbital manifestations concern the lacrimal gland (dacryoadenitis), extraocular muscles (orbital myositis), and soft tissues (orbital pseudotumor) [4, 10-13]. 
Common signs and symptoms of orbital GPA include proptosis, epiphora, diplopia, sudden onset of pain, erythema, eyelid edema and reduced vision. Proptosis is considered an important clinical sign in patients with suspected GPA, since in combination with upper or lower airway disease or glomerulonephritis, it implies a diagnosis of GPA. Proptosis and lid destruction may provoke exposure keratopathy, which can lead to corneal ulceration, ocular perforation, and blindness. Rarely, proptosis is followed by enophthalmos, which is a late consequence of chronic orbital inflammation due to fibrotic changes in the orbit. Epiphora is another symptom of orbital involvement that is often provoked by nasolacrimal duct obstruction or by lacrimal sac mucocele. Diplopia arises as a result of inflammation of extraocular muscles (orbital muscles), vasculitis of the vasa vasorum above, and compression of the optic nerve by the orbital mass, which may lead to optic nerve ischemia and even blindness. GPA may infiltrate the orbital apex and thus the optic nerve, resulting in painless optic neuropathy and edema of the optic nerve followed by atrophy. Further infiltration is responsible for painful ophthalmoplegia and blindness. Extension of the orbital mass leads to destruction of neighboring bony structures or neo-ossification. Note that orbital disease is associated with significant morbidity, including visual loss and facial deformity [10-13]. It is also worth mentioning that in the few cases of GPA in children, it initially presents with orbital manifestations, such as idiopathic inflammatory pseudotumor, proptosis secondary to orbital inflammation, and reduced vision [14].

\section{Eyelid, Lacrimal System, and Conjunctiva}

Eyelid involvement is uncommon in GPA, but may include dacryoadenitis, dacryocystitis, ptosis, lid granuloma, chalazion, entropion, trichiasis, and florid xanthelasma. Dacryoadenitis (inflammation of the lacrimal gland) is presented with pain and edema of the anterior orbit and of the eyelid, which impedes eye mobility. Dacryoadenitis and enlargement of the lacrimal gland may provoke ocular sicca syndrome $[4,12,15]$. Nasolacrimal duct obstruction is a late finding and can be either secondary to inflammatory spread from adjacent sinonasal disease, or a direct result of focal GPA inflammation. As a result of the nasolacrimal blockage, dacryocystitis and epiphora can occur. Florid xanthelasma (yellow lids) can appear even in patients with normal metabolism, and is a result of orbital inflammation in GPA, which may cause lipid accumulation $[16,17]$.

Conjunctival disease has been reported in up to $16 \%$ of patients with GPA. Early involvement is presented with conjunctival hyperemia. Granuloma ulceration and necrosis can be present, leading to cicatrizing conjunctivitis. Progressive conjunctival cicatrization can cause symblepharon or the formation of fibrovascular tissue that spreads across the ocular surface to the eyelid $[4,18,19]$. This results in entropion and trichiasis, with eyelashes turned against the globe. Conjunctival involvement can exhibit symptoms such as ocular redness, foreign body sensation, blurred vision, and possibly bloody tears [4]. Tarsal conjunctivitis, which can be associated with nasolacrimal duct obstruction and subglottic stenosis, has been also reported in patients with GPA [20].

\section{Sclera}

Scleritis is an inflammation of the whole thickness of the sclera, which can cause serious ocular morbidity with severe vision loss and morbidity. GPA can provoke nodular, diffuse, or necrotizing scleritis, which can be more severe than the scleritis of other etiologies [21]. The scleritis in GPA can be categorized into anterior or posterior, which have different complications. Anterior scleritis is also subcategorized into diffuse, nodular, or necrotizing. It is reported to occur in $16-38 \%$ of patients with GPA, making it the third most common ocular manifestation of GPA according to Hoffman et al. [22]. Scleritis can be the initial clinical manifestation of GPA, and typically presents with severe, deep, boring pain that can radiate to the temple and the jaw. The pain worsens at night, waking the patient up. It also manifests 
with eye redness and tenderness upon palpation $[23,24]$. Areas of severe vasculitis causing capillary occlusion in the deep episcleral vascular plexus characterize the disease. This situation leads to infarction and necrosis of the affected sclera, exposing the underlying pigmented uveal tissue of the choroid [22]. Necrotizing scleritis has been reported to follow routine cataract surgery in patients with GPA. It is characterized by scleral thinning and a bluish appearance of the choroid and is associated with systemic disease. It may result in scarring, infection and, in rare cases, ocular perforation and phthisis bulbi. Scleral inflammation can affect adjacent ocular structures such as the cornea, trabecular meshwork, and ciliary body, which can lead to keratitis, corneal ulceration, uveitis, ocular hypertension, or glaucoma [22-24].

\section{Cornea}

Corneal involvement is a common ocular manifestation of GPA. It can be either a primary or a secondary manifestation of the disease. The inflammation that characterizes GPA can specifically affect the cornea, resulting in peripheral ulcerative keratitis (PUK) $[25,26]$. PUK is usually unilateral and sectoral, but can also present as bilateral in up to $40 \%$ of patients, often in association with scleritis (mainly necrotizing). Patients commonly appear with ocular pain, injection, tearing, photophobia, and decreased vision. Histopathological findings of PUK reveal an immune-mediated occlusive necrotizing vasculitis of the anterior ciliary arteries that supply the sclera, the conjunctiva, and the peripheral cornea. PUK is often associated with scleritis as they have the same blood supply. In PUK, the lesions are situated within $2 \mathrm{~mm}$ of the corneoscleral limbus, while the adjacent limbal tissue and sclera have findings of vasculitic disease [26]. Collapse of the corneal epithelium makes the corneal stroma thinner, and if keratolysis progresses it can provoke perforation. Matrix metalloproteinases (MMPs) may participate in the observed keratolysis. PUK in GPA is also related to the presence of an autoantibody against cytokeratin
3 [27]. Apart from PUK, other corneal manifestations suggest exudative peripheral keratitis without ulceration, peripheral corneal thinning, and (rarely) interstitial keratitis [4, 28].

Corneal manifestations can also result from conjunctival or orbital involvement. Conjunctival cicatrization, accompanied by tear deficiency, entropion, trichiasis, and poor eyelid closure, can result in exposure keratopathy, secondary infection, and potential corneal perforation [18].

\section{Uvea}

Uveitis is a rare ocular manifestation of GPA, occurring in up to $10 \%$ of patients. It is categorized into anterior, intermediate, and posterior uveitis with varying degrees of vitritis, and it can be unilateral or bilateral. A granulomatous panuveitis has also been reported as an ocular manifestation of GPA. Uveitis usually accompanies keratitis or scleritis as a secondary manifestation, and sclerouveitis in particular probably worsens the ocular prognosis. The main symptoms of uveitis are blurred vision, floaters, photophobia, photopsia, and redness of the eye [4].

\section{Retina and Choroid}

Retinal and choroidal manifestations rarely occur in GPA, with vessel involvement being most common. The retinal and choroidal circulation of the eye can be affected with or without clear signs of vasculitis. This involvement can be unilateral or bilateral, central or multifocal [4].

Retinal vein occlusion can be present without findings of vasculitis. It can be a result of compression of the vessels from external tissues [29]. On the other hand, retinal artery occlusion is usually associated with vasculitis [30]. Retinal and choroid manifestations that can be recognized in GPA are retinitis, chorioretinitis, macular edema, exudative retinal detachment, and retinal necrosis [4, 31-33]. Sclerochoroidal granulomas may also appear as uveal melanoma [4]. Other unusual manifestations of GPA include vitreous hemorrhages due to 
chorioretinal and ciliary body granulomas, and acute multifocal placoid pigment epitheliopathy [4].. The usual findings upon clinical examination are perivascular sheathing, focal arterial or venous infarction, and retinal ischemia, presenting as white or creamy lesions at the level of the retinal pigment epithelium, choroidal folds, cotton-wool exudates, retinal neovascularization, and choroidal thickening [4].

\section{Neuro-ophthalmologic Manifestations}

Neuro-ophthalmologic manifestations in GPA may be secondary to the formation of granulomas or to the vasculitis that appears in the disease. Orbital inflammatory disease and granulomas can lead to diplopia or loss of vision due to compressive optic neuropathy. This neuropathy is a result of the infiltration of the adjacent tissues by the orbital mass. Additionally, vasculitis can interrupt the blood supply of the optic and other cranial nerves, causing similar findings [4].

Furthermore, cases of vision loss as a result of optic neuritis or perineuritis due to GPA have been reported in the context of the extension of granulomatous inflammation [34-38]. Oculomotor nerves can also be affected by the vasculitis in GPA, provoking palsy of the oculomotor, trochlear, and abducens nerve [39]. Horner's syndrome rarely appears in patients with GPA [4]. Patients with neuro-ophthalmic disease usually present with diplopia, reduced visual acuity or sudden visual loss, an afferent pupillary defect, and visual field loss that can be sectoral [34-38].

\section{DIAGNOSIS}

Ocular disease may be the dominant manifestation in patients with GPA. Thus, an ophthalmologist should have a high index of suspicion, especially in cases where other features of the disease, such as pulmonary or renal disease, are absent [40]. The ANCA test is recognized to be sensitive and specific for GPA, especially for the systemic form of the disease, as it is considered to be an indicator of the activity of the disease.
The majority (80-95\%) of patients are associated with c-ANCA against proteinase 3 (PR3); the remaining cases are associated with p-ANCA against myeloperoxidase (MPO). In patients with the limited form of GPA, ANCA are found in approximately 50-60\% of patients [4].

Diagnostic imaging methods such as computed tomography (CT), magnetic resonance imaging (MRI), and ultrasound have diagnostic value in cases of orbital involvement, demonstrating orbital lesions, involvement of adjacent structures, and the extent of tissue damage, thus allowing the response to medical treatment to be determined. CT offers the ability to depict sinus structure disorders and osseous invasion, while MRI is helpful for identifying granulomas and delineating orbital mucosal changes [1]. A diagnosis of retinal or vascular involvement in GPA is often confirmed by means of fluorescent angiography, which depicts delayed or absent filling of the blood vessels or leakage in areas of ischemia [4].

However, imaging findings are not always specific for GPA. In these cases, biopsy can confirm the diagnosis, especially for orbital GPA and conjunctival involvement. Classical histologic features of GPA (granulomas, necrosis, vasculitis) are not always present in the biopsy, mainly in cases of ocular GPA. Therefore, one histologic finding that is useful in the diagnosis of GPA over other orbital inflammatory diseases is the presence of scattered eosinophils admixed within formed granulomas [41].

\section{TREATMENT}

Before the arrival of immunomodulatory therapy, GPA was a fatal disease with a bad prognosis with a median survival of 5 months. Currently, the combination of glucocorticoids and cytotoxic agents has changed the prognosis of GPA patients, with $95 \%$ survival at 5 years and $80 \%$ survival at 10 years.

Regarding the ocular manifestations of GPA, localized pharmaceutical and surgical treatment may be helpful to achieve remission. Topical corticosteroid therapy may be useful for managing non-vision-threatening ocular manifestations of GPA such as episcleritis, 
conjunctivitis, and mild anterior uveitis. Complications of the above situations, such as necrotizing scleritis, posterior scleritis, PUK, bilateral ocular involvement, orbital or adnexal manifestations, or retinal and optic nerve vasculitis may demand aggressive systematic immunosuppressive treatment with corticosteroids and cyclosporine $[4,41]$. Therefore, conventional treatment for severe forms of the disease inlude high-dose corticosteroids and cyclophosphamide for at least 3 months (initial treatment) until remission, then treatment with azathioprine or methotrexate for a period of at least 2 years (maintenance therapy) [42].

However, increased understanding of the mechanisms involved in the inflammatory response and the regulation of adaptive immunity led to the development of biotherapeutics, including monoclonal antibodies. Specifically, several studies have shown that anti-tumor necrosis factor (anti-TNF) antibodies (i.e., infliximab) could be useful in the management of non-infectious scleritis secondary to GPA $[42$, 43]. Additionally, rituximab (a chimeric monoclonal anti-CD20) has been found to be safe and effective for controlling refractory PUK, scleritis, and uveitis associated with GPA, with eventual progression towards steroid-sparing remission [42, 44-47].

Surgical management has limited effectiveness, as there is a possibility of relapse, although it is helpful in severe situations. In cases of nasolacrimal duct obstruction, dacryocystorhinostomy with the creation of a new outflow to bypass the obstruction may be required $[4,21]$. Moreover, PUK and necrotizing scleritis with impending ocular perforation demand more invasive methods such as conjunctival resection and tectonic corneal grafting [4, 19]. Severe ocular proptosis and optic nerve compression due to granulomas may require orbital decompression [4]. It is suggested that no surgery should be performed during active disease and strict control of the disease is necessary in the postoperative period. In any case, appropriate multidisciplinary intervention is required so as to reduce the relapse and the morbidity of these patients.

\section{ACKNOWLEDGEMENTS}

Funding. No funding or sponsorship was received for this study or the publication of this article.

Authorship. All named authors meet the International Committee of Medical Journal Editors (ICMJE) criteria for authorship for this manuscript, take responsibility for the integrity of the work as a whole, and have given final approval to the version to be published.

Disclosures. Evdokia Sfiniadaki, Ioanna Tsiara, Panagiotis Theodossiadis, and Irini Chatziralli have nothing to disclose.

Compliance with Ethics Guidelines. This article is based on previously conducted studies and does not contain any studies with human participants or animals performed by any of the authors.

Open Access. This article is distributed under the terms of the Creative Commons Attribution-NonCommercial 4.0 International License (http://creativecommons.org/licenses/ by-nc/4.0/), which permits any noncommercial use, distribution, and reproduction in any medium, provided you give appropriate credit to the original author(s) and the source, provide a link to the Creative Commons license, and indicate if changes were made.

\section{REFERENCES}

1. Catanoso M, Macchioni P, Boiardi L, et al. Epidemiology of granulomatosis with polyangiitis (Wegener's granulomatosis) in Northern Italy: a 15-year population-based study. Semin Arthritis Rheum. 2014;44(2):202-7.

2. Greco A, Marinelli C, Fusconi $M$, et al. Clinic manifestations in granulomatosis with polyangiitis. Int J Immunopathol Pharmacol. 2016;29(2):151-9.

3. Comarmond C, Cacoub P. Granulomatosis with polyangiitis (Wegener): clinical aspects and treatment. Autoimmun Rev. 2014;13(11):1121-5. 
4. Tarabishy AB, Schulte M, Papaliodis GN, Hoffman GS. Wegener's granulomatosis: clinical manifestations, differential diagnosis, and management of ocular and systemic disease. Surv Ophthalmol. 2010;55(5):429-44.

5. Csernok E, Gross WL. Current understanding of the pathogenesis of granulomatosis with polyangiitis (Wegener's). Expert Rev Clin Immunol. 2013;9(7): 641-8.

6. Xie G, Roshandel D, Sherva R, et al. Association of granulomatosis with polyangiitis (Wegener's) with HLA-DPB $1{ }^{*} 04$ and SEMA6A gene variants: evidence from genome-wide analysis. Arthritis Rheumatol. 2013;65(9):2457-68.

7. Martorana D, Maritati F, Malerba G, et al. PTPN22 R620W polymorphism in the ANCA-associated vasculitides. Rheumatology. 2012;51(5):805-12.

8. Hadzik-Blaszczyk M, Zdral A, Zielonka TM, et al. SERPINA1 gene variants in granulomatosis with polyangiitis. Adv Exp Med Biol. 2018;1070:9-18.

9. Iannella G, Greco A, Granata G, et al. Granulomatosis with polyangiitis and facial palsy: literature review and insight in the autoimmune pathogenesis. Autoimmun Rev. 2016;15(7):621-31.

10. Chipczyńska B, Grałek M, Hautz W, et al. Orbital tumor as an initial manifestation of Wegener's granulomatosis in children: a series of four cases. Med Sci Monit. 2009;15(8):CS135-8.

11. Lopes Caçola R, Morais SA, Carvalho R, Môço R. Bilateral dacryoadenitis as initial presentation of a locally aggressive and unresponsive limited form of orbital granulomatosis with polyangiitis. BMJ Case Rep. 2016. https://doi.org/10.1136/bcr-2015-214099.

12. Hibino M, Kondo T. Dacryoadenitis with ptosis and diplopia as the initial presentation of granulomatosis with polyangiitis. Intern Med. 2017;56(19): 2649-53.

13. Muller K, Lin JH. Orbital granulomatosis with polyangiitis (Wegener granulomatosis): clinical and pathologic findings. Arch Pathol Lab Med. 2014;138(8):1110-4.

14. Ure E, Kayadibi Y, Sanli DT, Hasiloglu ZI. Orbital involvement as the initial presentation of Wegener granulomatosis in a 9-year-old girl: MR imaging findings. Diagn Interv Imaging. 2016;97(11): 1181-2.

15. Ghanem RC, Chang N, Aoki L, Santo RM, Matayoshi S. Vasculitis of the lacrimal sac wall in Wegener granulomatosis. Ophthalmic Plast Reconstr Surg. 2004;20(3):254-7.
16. Hello M, Barbarot S, Masseau A, Cassagnau E, Hamidou M. Xanthelasma associated with Wegener's granulomatosis. Ann Dermatol Venereol. 2010;137(2):107-10.

17. Tullo AB, Durrington $\mathrm{P}$, Graham E, et al. Florid xanthelasmata (yellow lids) in orbital Wegener's granulomatosis. Br J Ophthalmol. 1995;79(5):453-6.

18. Jordan DR, Zafar A, Brownstein S, Faraji H. Cicatricial conjunctival inflammation with trichiasis as the presenting feature of Wegener granulomatosis. Ophthalmic Plast Reconstr Surg. 2006;22(1):69-71.

19. Miserocchi E, Waheed NK, Baltatzis S, Foster CS. Chronic cicatrizing conjunctivitis in a patient with ocular cicatricial pemphigoid and fatal Wegener granulomatosis. Am J Ophthalmol. 2001;132(6): 923-4.

20. Robinson MR, Lee SS, Sneller MC, Lerner R, Langford CA, Talar-Williams C, Cox TA, Chan CC, Smith JA. Tarsal-conjunctival disease associated with Wegener's granulomatosis. Ophthalmology. 2003;110(9):1770-80.

21. Sadiq SA, Jennings CR, Jones NS, Downes RN. Wegener's granulomatosis: the ocular manifestations revisited. Orbit. 2000;19(4):253-61.

22. Hoffman GS, Kerr GS, Leavitt RY, et al. Wegener granulomatosis: an analysis of 158 patients. Ann Intern Med. 1992;116(6):488-98.

23. Cocho L, Gonzalez-Gonzalez LA, Molina-Prat N, Doctor P, Sainz-de-la-Maza M, Foster CS. Scleritis in patients with granulomatosis with polyangiitis (Wegener). Br J Ophthalmol. 2016;100(8):1062-5.

24. Arnavaz AB, Freissler K, Lang GE. Posterior scleritis in Wegener disease. Ophthalmologe. 1997;94(8):595-9.

25. Fortney AC, Chodosh J. Conjunctival ulceration in recurrent Wegener granulomatosis. Cornea. $2002 ; 21(6): 623-4$.

26. Cao Y, Zhang W, Wu J, Zhang H, Zhou H. Peripheral ulcerative keratitis associated with autoimmune disease: pathogenesis and treatment. J Ophthalmol. 2017;2017:7298026.

27. Reynolds I, Tullo AB, John SL, Holt PJ, Hillarby MC. Corneal epithelial-specific cytokeratin 3 is an autoantigen in Wegener's granulomatosis-associated peripheral ulcerative keratitis. Investig Ophthalmol Vis Sci. 1999;40(9):2147-51.

28. Reddy SC, Tajunisah I, Rohana T. Bilateral scleromalacia perforans and peripheral corneal thinning in Wegener's granulomatosis. Int J Ophthalmol. 2011;4(4):439-42. 
29. Wang M, Khurana RN, Sadda SR. Central retinal vein occlusion in Wegener's granulomatosis without retinal vasculitis. $\mathrm{Br} \mathrm{J}$ Ophthalmol. 2006;90(11):1435-6.

30. Lozano-López V, Rodríguez-Lozano B, Losada-Castillo MJ, Delgado-Frías E, Dopazo-Luque D, SerranoGarcía M. Central retinal artery occlusion in Wegener's granulomatosis: a diagnostic dilemma. J Ophthalmic Inflamm Infect. 2011;1(2):71-5.

31. Proia AD. Granulomatous choroiditis in Wegener granulomatosis. Arch Ophthalmol. 2011;129(4): 520-1.

32. Lim LT, Vasudevan V, Shelton J, Vitale AT, Moorthy R. Macula retinal vasculitis and choroiditis associated with granulomatosis with polyangiitis. Retin Cases Brief Rep. 2017;11(2):119-22.

33. Jaben SL, Norton EW. Exudative retinal detachment in Wegener's granulomatosis: case report. Ann Ophthalmol. 1982;14(8):717-20.

34. Takazawa $\mathrm{T}$, Ikeda $\mathrm{K}$, Nagaoka $\mathrm{T}$, et al. Wegener granulomatosis-associated optic perineuritis. Orbit. 2014;33(1):13-6.

35. Purvin V, Kawasaki A. Optic perineuritis secondary to Wegener's granulomatosis. Clin Exp Ophthalmol. 2009;37(7):712-7.

36. Moubayed SP, Black DO. Optic neuritis as an initial presentation of Wegener's granulomatosis. Can J Ophthalmol. 2009;44(6):e59.

37. Niskopoulou M, Du Toit N. Optic neuritis as a feature of Wegener's granulomatosis. Eye. 2002;16(3): 320-1.

38. Monteiro ML, Borges WI, do Val Ferreira Ramos C, Lucato LT, Leite CC. Bilateral optic neuritis in Wegener granulomatosis. J Neuroophthalmol. 2005;25(1):25-8.

39. Nowack R, Wachtler P, Kunz J, Rasmussen N. Cranial nerve palsy in Wegener's granulomatosis- lessons from clinical cases. J Neurol. 2009;256(3): 299-304.

40. Garrity JA. Ocular manifestations of small-vessel vasculitis. Cleve Clin J Med. 2012;79(Suppl 3):S31-3.

41. Kubaisi B, Abu Samra K, Foster CS. Granulomatosis with polyangiitis (Wegener's disease): an updated review of ocular disease manifestations. Intractable Rare Dis Res. 2016;5(2):61-9.

42. Saadoun D, Bodaghi B, Bienvenu B, Wechsler B, Sene D, Trad S, Abad S, Cacoub P, Kodjikian L, Sève $P$. Biotherapies in inflammatory ocular disorders: interferons, immunoglobulins, monoclonal antibodies. Autoimmun Rev. 2013;12(7):774-83.

43. Doctor P, Sultan A, Syed S, Christen W, Bhat P, Foster CS. Infliximab for the treatment of refractory scleritis. Br J Ophthalmol. 2010;94(5):579-83.

44. You C, Ma L, Lasave AF, Foster CS. Rituximab induction and maintenance treatment in patients with scleritis and granulomatosis with polyangiitis (Wegener's). Ocul Immunol Inflamm. 2018;26(8): 1166-73.

45. Huerva V, Sanchez MC, Traveset A, Jurjo C, Ruiz A. Rituximab for peripheral ulcerative keratitis with Wegener granulomatosis. Cornea. 2010;29(6): 708-10.

46. Ahmed A, Foster CS. Cyclophosphamide or rituximab treatment of scleritis and uveitis for patients with granulomatosis with polyangiitis. Ophthalmic Res. 2019;61(1):44-50.

47. Joshi L, Tanna A, McAdoo SP, Medjeral-Thomas N, Taylor SR, Sandhu G, Tarzi RM, Pusey CD, Lightman S. Long-term outcomes of rituximab therapy in ocular granulomatosis with polyangiitis: impact on localized and nonlocalized disease. Ophthalmology. 2015;122(6):1262-8. 